\title{
IVF booster offered in Canada but not US
}

$\mathrm{A}$ new fertility treatment that purports to help older women get pregnant by boosting the energy in their eggs is now being offered in Toronto but nowhere else in North America. A trial in the US was voluntarily suspended after the Food and Drug Administration suggested the treatment may be considered an "investigational new drug" requiring approval. In Canada, it may fall outside regulation, though Health Canada has not made any formal response to date.

The treatment, called Augment, involves extracting mitochondria, a cell's energy supply, from a woman's ovarian stem cells and injecting them into her eggs during in vitro fertilization. In December 2014, OvaScience, the US-based company that developed Augment, announced that the treatment would be offered commercially. No clinical data on efficacy were provided, though the company noted that 150 women had been enrolled in experimental trials at four sites worldwide. These include the Toronto Centre for Advanced Reproductive Technology (TCART) and clinics in London, Istanbul and Dubai. OvaScience Chief Executive Officer Michelle Dipp said they expected Augment would be used in approximately 1000 paying patients in 2015 .

Underactive mitochondria are thought to be a key reason why older eggs fail to develop into blastocysts embryos that are ready to implant. Augment adds extra, and apparently less mutated, mitochondria. The researchers think the additional mitochondria provide the energy needed for successful cell division. Research in pigs has shown that adding mitochondria leads to higher rates of pregnancy. Research in humans also suggested that mitochondria borrowed from young donated eggs increased chances of pregnancy, but the idea of using another woman's mitochondria raised safety concerns, and a moratorium was placed on the practice more than a decade ago.

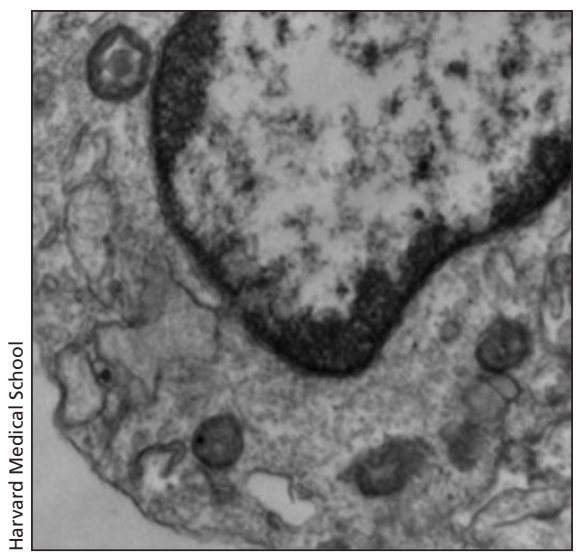

A new treatment boosts the energy in eggs by extracting mitochondria (depicted) from a woman's ovarian stem cells and injecting them into her eggs during in vitro fertilization.

Augment gets around that problem by using the would-be mother's own mitochondria. This only became possible after Jonathan Tilly, a researcher then at Harvard Medical School, discovered that the adult human ovary contained stem cells, capable of giving rise to fully mature oocytes. Though Tilly had previously identified them in mice, it was the discovery in humans that shook things up, overturning the decades-old belief that human females are born with all the eggs they will ever possess. Tilly and colleagues founded OvaScience, which is dedicated to developing fertility treatments based on ovarian stem cells.

Augment requires a woman to undergo an ovarian biopsy, during which pieces of her ovary's outer layer, where ovarian stem cells reside, are removed. After the stem cells are harvested, they are counted and frozen. On the day of egg retrieval, the stem cells are thawed and the mitochondria are isolated, prepared and then injected into the eggs along with sperm.

The Augment lab in Toronto, located within TCART premises, was built and is operated by OvaScience, whose technicians carry out the proprietary process. Dr. Robert Casper, medical director of TCART and a professor at the University of Toronto, says the clinic has enrolled 60 Canadian patients into the trial, and by the end of 2014 , 15 of them had completed their cycles.

"It's looking promising," says Casper, who declined to provide specific numbers. "We're getting blastocysts in people who didn't get blastocysts before, and we've got some pregnancies already. We're very encouraged." Casper, who is also a senior investigator at Mount Sinai Hospital's LunenfeldTanenbaum Research Institute, has also carried out extensive research on mitochondria.

Trial participants did not pay for any part of the treatment, says Casper. But he estimates that Augment will add about $\$ 25000$ to the cost of an IVF cycle. Most of that cost is set by OvaScience, which charges clinics a fixed fee for each cycle. Many of the Augment patients at TCART in 2015 are expected to be from the US.

In September 2013, OvaScience, based in Boston, voluntarily suspended its US trial after receiving a notice from the FDA. OvaScience had assumed it did not need regulatory approval, since the process involved injecting a woman's own organelles into her own egg using a method already used to deliver sperm. Neither the FDA nor the company would comment on the status of Augment in the US, but the company has now announced it will be establishing its international headquarters in the UK.

Ubaka Ogbogu, a professor in the faculties of Law and Pharmacy \& Pharmaceutical Sciences at the University of Alberta in Edmonton, notes that the FDA and Health Canada differ in their approach to medical treatments.

"Health Canada has taken the position that they don't regulate treatments," he says. But he believes that is problematic: "At a minimum, this is an experiment and needs regulatory scrutiny. To regard it as a treatment procedure is dishonest."

Dr. Arthur Leader, cofounder of the 
Ottawa Fertility Centre and a former member of the stem cell oversight committee of the Canadian Institutes of Health Research, says his clinic was approached by OvaScience about offering the treatment, but his team declined to pursue it, given the lack of data and regulatory oversight. "We weren't comfortable with it."
"I do think there are important questions to ask about the children who result," says Marcy Darnovsky, executive director of the Center for Genetics and Society, an advocacy group based in Berkeley, California. "I sure would want to see a clinical trial."

Arthur Caplan, a bioethicist at New
York University Langone Medical Center in New York City agrees: "You're innovating in an area where the burden of a bad outcome goes on for a lifetime and the person bearing the highest cost can't consent." - Alison Motluk, Toronto, Ont.

CMAJ 2015. DOI:10.1503/cmaj.109-4975 